\title{
Atividade hepatoprotetora dos extratos etanólico e hexânico das folhas de Momordica charantia L.
}

PEREIRA, B.S. ${ }^{1}$; NUNES-PINHEIRO, D.C.S. ${ }^{1 *}$; VASCONCELOS, A.K.P. ; PINHEIRO, A.D.N. ${ }^{2}$; RODRIGUES, P.A. ${ }^{1}$ ${ }^{1}$ Programa de Pós-Graduação em Ciências Veterinárias da Universidade Estadual do Ceará (UECE), Avenida Paranjana, 1700, Campus do Itaperi, Serrinha, CEP: 60740-000, Fortaleza-Brasil * csnpdiana@hotmail.com ${ }^{2}$ Curso de Farmácia do Centro de Ciências da Saúde da Universidade Federal do Ceará, Rua Alexandre Baraúna, 949, Bairro Rodolfo Teófilo, CEP: 60430-160,Fortaleza-Brasil anadebora@gmail.com

RESUMO: Momordica charantia L. (Cucurbitaceae) é empregada na medicina popular pelas diversas propriedades biológicas e farmacológicas. Neste trabalho, objetivou-se avaliar a atividade hepatoprotetora dos extratos hexânico (EH) e etanólico (EE) das folhas de $M$. charantia no modelo de lesão hepática aguda induzida pelo etanol. Em ambos os extratos foram detectados esteróides; EH apresentou atividade antioxidante mais marcante que EE. O pré-tratamento de camundongos (Swiss, machos) por via oral por três dias consecutivos com 25, 50 ou $100 \mathrm{mg} \mathrm{Kg}^{-1}$ de EH preveniu o aumento tanto de AST quanto de ALT $(p<0,05)$ enquanto o pré-tratamento com EE preveniu apenas o aumento de ALT $(p<0,05)$. Ambos os extratos reduziram a atividade das enzimas hepáticas, contudo EH apresentou efeito mais relevante, caracterizando potencial hepatoprotetor, possivelmente associado ao seu potencial antioxidante.

Palavras-chave: Momordica charantia, Cucurbitaceae, biomarcadores, atividade hepática, etanol

\begin{abstract}
Hepatoprotective activity of hexane and ethanol extracts from Momordica charantia L. leaves. Momordica charantia L. (Cucurbitaceae) has been used in folk medicine based on its several biological and pharmacological properties. The aim of this work was to evaluate the hepatoprotective activity of hexane $(\mathrm{HE})$ and ethanol $(\mathrm{EE})$ extracts from $M$. charantia leaves on the acute liver injury model induced by ethanol. Steroids were detected in both extracts, and $\mathrm{EH}$ had higher antioxidant activity than EE. Oral pretreatment of male Swiss mice for three consecutive days with 25,50 or $100 \mathrm{mg} \mathrm{kg}^{-1} \mathrm{HE}$ prevented the increase in AST and ALT $(p<0.05)$, whereas pretreatment with EE only prevented ALT increase $(p<0.05)$. Both extracts reduced the activity of hepatic enzymes; however, $\mathrm{HE}$ had a more relevant effect, characterizing hepatoprotective potential, likely associated with its antioxidant potential.
\end{abstract}

Key words: Momordica charantia, Cucurbitaceae, biomarkers, hepatic activity, ethanol

\section{INTRODUÇÃO}

Momordica charantia L. (Cucurbitaceae) é encontrada em abundância no Nordeste brasileiro e as folhas são utilizadas na forma de infusão e cozimento, como antidiarréico e antirreumático (Pramyothin et al., 2006). Esta planta é amplamente estudada e possui diversas propriedades farmacológicas. $O$ extrato etanólico obtido das folhas apresentou atividades anti-helmíntica contra Haemonchus contortus (Batista et al., 1999), antiinflamatória (Farias, 2003), antifúngica em modelo de dermatofitose provocado por Microsporum canis (Braga et al., 2007), cicatrizante (Vasconcelos et al., 2007) e gastroprotetora (Leite et al., 2002). Os extratos das folhas (aquoso, etanólico e metanólico) apresentaram, também, atividade antimicrobiana (Khan, 1998). Os frutos de $M$. charantia são bastante estudados pelas propriedades hipoglicemiantes (Kumar et al., 2008; Kumar et al., 2009). Os extratos etanólico (Gürbüz et al., 2000) e metanólico (Alam et al., 2009) obtidos dos frutos demonstraram ser antiulceratogênicos, no entanto o extrato etanólico mostrou-se hepatotóxico (El-Batran et al., 2006). Os extratos dos frutos não apresentaram nefrotoxicidade e nem hepatotoxicidade em baixas doses, além de não alterarem o consumo de alimentos, o ganho de peso ou os parâmetros hematológicos (Platel et al., 1993; Virdi et al., 2003).

Recebido para publicação em 28/02/2009

Aceito para publicação em 18/04/2010

Rev. Bras. PI. Med., Botucatu, v.12, n.3, p.311-316, 2010. 
As doenças hepáticas são consideradas um dos maiores problemas de saúde, sendo o fígado um órgão importante tanto na detoxicação como deposição de substâncias endógenas e exógenas (Sehrawat et al., 2006). Dentre estas substâncias, os efeitos deletérios do álcool sobre este órgão são conhecidos e estão associados a várias doenças. Apesar das várias propriedades biológicas de $M$. charantia não foram encontrados dados na literatura do efeito das folhas sobre a função hepática. Sendo assim, o presente trabalho teve por objetivo avaliar a atividade hepatoprotetora dos extratos etanólico e hexânico das folhas de M. charantia L., em modelo de lesão aguda induzida por etanol.

\section{MATERIAL E MÉTODO}

Preparo dos extratos e estudo fitoquímico M. charantia foi coletada pela manhã no mês de março de 2005, nas proximidades do Aeroporto Internacional de Fortaleza, Fortaleza, Ceará. A planta foi identificada no Herbário Prisco Bezerra do Departamento de Botânica e Biologia da Universidade Federal do Ceará (UFC), onde foi depositada, recebendo o voucher de número 32441.

Os extratos hexânico e etanólico foram preparados a partir das folhas de $M$. charantia. Para o preparo do extrato hexânico, as folhas secas foram submersas em hexano por sete dias. A solução obtida foi filtrada e evaporada a $69^{\circ} \mathrm{C}$, obtendo-se o extrato hexânico (EH). O resíduo do material que ficou exposto ao hexano foi mantido em ambiente arejado por três dias para evaporação do hexano residual e, posteriormente, foi submerso em etanol por sete dias, para obtenção do extrato etanólico (EE), que após filtração foi evaporado a $78,5^{\circ} \mathrm{C}$. Os extratos obtidos, EH e EE, foram diluídos em solução de $\mathrm{NaCl} 0,9 \%$ acrescida de $0,5 \%$ de Tween 20 e administrados aos camundongos por via oral nas concentrações de 25,50 ou $100 \mathrm{mg} \mathrm{Kg}^{-1}$ de acordo com o protocolo experimental.

Os testes fitoquímicos foram realizados para identificar fenóis, taninos, leucoantocianidinas, catequinas, flavonas, flavonóis, flavononas, flavanonóis, xantonas, triterpenóides, saponinas, resinas, esteróides e alcalóides (Matos, 1997).

\section{Determinação da atividade antioxidante in vitro}

Para a avaliação da atividade antioxidante foi utilizado o método de varredura de radical livre, DPPH (1,1-difenil-2-picrihidrazil) (Bondet et al., 1997). Nesta técnica, a solução fortemente corada de um radical livre foi tratada com solução do composto químico. Em tubo de ensaio foram colocados $3,9 \mathrm{~mL}$ de solução do radical livre DPPH $\left(6,5 \times 10^{-5} \mathrm{M}\right)$ em metanol e em seguida foi adicionado $0,1 \mathrm{~mL}$ da solução etanólica ou hexânica $(2.000,5.000$ ou 10.000 ppm) e a absorbância máxima foi medida a $515 \mathrm{~nm}$ durante uma hora. O teste foi realizado em triplicata e os resultados foram expressos em índice de varredura de radicais livres (IV\%).

\section{Animais}

Camundongos Swiss, machos, entre 2 e 4 meses, oriundos do Biotério Central da Universidade Federal do Ceará, foram mantidos na sala de experimentação animal do Laboratório de Imunologia e Bioquímica (LIBA/FAVET) da Universidade Estadual do Ceará em caixas plásticas sob condições adequadas de luz e temperatura, recebendo ração e água à vontade. Os animais foram separados em grupos, mantidos em caixas plásticas assépticas, e foram manuseados de acordo com as normas éticas da experimentação animal. Antes de cada experimento os animais foram mantidos em jejum de 18 horas recebendo água a vontade.

O uso de animais no protocolo de pesquisa foi conduzido segundo as normas do Colégio Brasileiro de Experimentação Animal (COBEA) e este protocolo foi previamente aprovado pelo Comitê de Ética em Pesquisa da Universidade Estadual do Ceará, protocolo número 04185875-1.

\section{Efeito do pré-tratamento com os extratos hexânico e etanólico sobre marcadores enzimáticos séricos de hepatotoxicidade}

Os biomarcadores séricos, que avaliam a atividade hepática, foram medidos utilizando-se as atividades das enzimas alanina aminotransferase (AST), aspartato aminotransferase (ALT) e lactato desidrogenase (LDH).

O sangue dos animais foi coletado com 0 auxílio de capilar pelo plexo retro-orbital antes de iniciar os tratamentos (D0) e no terceiro dia (D3) após o final dos tratamentos e indução de lesão por etanol. A lesão hepática foi induzida pela administração de $0,2 \mathrm{~mL}$ do agente indutor, etanol $95 \%$ (Sairam et al., 2002). Ao final do experimento os animais foram sacrificados. Para a determinação dos valores séricos das enzimas foi utilizado o soro individual dos animais de cada grupo, seguindo as instruções dos fabricantes dos Kits comerciais (Wiener®) para aparelho de automação (BT plus ${ }^{\circledR}$ ). As dosagens de AST, ALT e LDH foram expressas em unidades

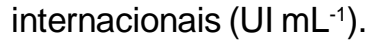

Todos os soros foram congelados a $-20^{\circ} \mathrm{C}$ por no máximo três dias e ao serem descongelados não mais foram utilizados.

\section{Análise estatística}

Os resultados das dosagens séricas de AST, ALT e LDH foram expressos como média e desvio padrão e analisados por ANOVA seguido do teste Tukey $(p<0,05)$. 


\section{RESULTADO}

Análise fitoquímica dos extratos

Os testes fitoquímicos utilizados identificaram a presença de esteróides em ambos os extratos.

\section{Atividade antioxidante}

EE das folhas de $M$. charantia nas doses de 2, 5 e $10 \mathrm{mg} \mathrm{mL}^{-1}$ apresentou índice de varredura (IV) de 28,$66 ; 38,73$ e $39,75 \%$, respectivamente. EH das folhas de $M$. charantia nas doses de 2,5 e $10 \mathrm{mg}$ $\mathrm{mL}^{-1}$ apresentou IV de 40,07; 76,64 e 79,79\%, respectivamente.

\section{Lesão hepática induzida pelo etanol}

O grupo de animais que recebeu somente etanol (grupo etanol) apresentou elevação significativa nos níveis séricos de ALT e AST $(p<0,05)$, mas não alterou os níveis de LDH em relação aos grupos salina e Tween não tratados com etanol (Tabelas 1, 2 e 3).

TABELA 1. Níveis séricos da enzima aspartato aminotransferase (AST, UI $\mathrm{mL}^{-1}$ ) no inicio (D0) e final (D3) dos tratamentos com os extratos hexânico $(\mathrm{EH})$ e etanólico $(\mathrm{EE})$ das folhas de $M$. charantia sobre a atividade hepática.

\begin{tabular}{lcccc}
\hline Grupos & Dose $\left(\mathbf{m L}\right.$ ou $\left.\mathbf{~ m ~ k ~}^{-1}\right)$ & Número de animais & D0 & D3 \\
\hline Salina & 0,2 & 10 & $78,33 \pm 23,02^{\mathrm{a}}$ & $80,63 \pm 21,97^{\mathrm{a}, \mathrm{A}}$ \\
Tween & 0,2 & 10 & $87,10 \pm 23,60^{\mathrm{a}}$ & $90,30 \pm 16,15^{\mathrm{a}, \mathrm{A}}$ \\
Etanol & 0,2 & 10 & $94,80 \pm 25,90^{\mathrm{a}}$ & $142,12 \pm 51,74^{\mathrm{b}, \mathrm{B}}$ \\
EE & 25 & 05 & $72,42 \pm 16,60^{\mathrm{a}}$ & $109,00 \pm 10,95^{\mathrm{a}, \mathrm{A}}$ \\
EE & 50 & 05 & $84,66 \pm 15,26^{\mathrm{a}}$ & $79,20 \pm 12,53^{\mathrm{a}, \mathrm{A}}$ \\
EE & 100 & 05 & $71,42 \pm 12,56^{\mathrm{a}}$ & $98,00 \pm 10,41^{\mathrm{a}, \mathrm{A}}$ \\
EE + Etanol & 25 & 10 & $72,42 \pm 16,60^{\mathrm{a}}$ & $201,42 \pm 53,41^{\mathrm{b}, \mathrm{C}}$ \\
EE + Etanol & 50 & 10 & $84,66 \pm 15,26^{\mathrm{a}}$ & $155,92 \pm 46,03^{\mathrm{b}, \mathrm{B}}$ \\
EE + Etanol & 100 & 10 & $71,42 \pm 12,56^{\mathrm{a}}$ & $119,61 \pm 33,82^{\mathrm{b}, \mathrm{B}}$ \\
EH & 25 & 05 & $78,50 \pm 33,37^{\mathrm{a}}$ & $90,00 \pm 11,91^{\mathrm{a}, \mathrm{A}}$ \\
EH & 50 & 05 & $73,00 \pm 14,76^{\mathrm{a}}$ & $113,0 \pm 22,92^{\mathrm{a}, \mathrm{A}}$ \\
EH & 100 & 05 & $90,50 \pm 17,91^{\mathrm{a}}$ & $116,0 \pm 21,00^{\mathrm{a}, \mathrm{A}}$ \\
EH + Etanol & 25 & 10 & $78,50 \pm 33,37^{\mathrm{a}}$ & $79,77 \pm 20,58^{\mathrm{a}, \mathrm{A}}$ \\
EH + Etanol & 50 & 10 & $73,00 \pm 14,76^{\mathrm{a}}$ & $75,30 \pm 12,24^{\mathrm{a}, \mathrm{A}}$ \\
EH + Etanol & 100 & 10 & $90,50 \pm 17,91^{\mathrm{a}}$ & $76,30 \pm 21,44^{\mathrm{a}, \mathrm{A}}$ \\
\hline
\end{tabular}

Letras diferentes diferem significativamente entre si $(p<0,05)$. Letras minúsculas comparam D0 com D3 na mesma linha, e letras maiúsculas comparam os tratamentos em D3, na mesma coluna.

TABELA 2. Níveis séricos da enzima alanina aminotransferase (ALT, $U I \mathrm{~mL}^{-1}$ ) no inicio (D0) e final (D3) dos tratamentos com os extratos hexânico $(\mathrm{EH})$ e etanólico (EE) das folhas de $M$. charantia sobre a atividade hepática.

\begin{tabular}{lcccc}
\hline Grupos & Dose $\left(\mathbf{m L}\right.$ ou $\left.\mathbf{~ m ~ k g ~}^{-1}\right)$ & Número de animais & D & D3 \\
\hline Salina & 0,2 & 10 & $63,60 \pm 17,44^{\mathrm{a}}$ & $50,27 \pm 10,86^{\mathrm{a}, \mathrm{A}}$ \\
Tween & 0,2 & 10 & $55,40 \pm 12,50^{\mathrm{a}}$ & $58,12 \pm 17,70^{\mathrm{a}, \mathrm{A}}$ \\
Etanol & 0,2 & 10 & $61,70 \pm 16,15^{\mathrm{a}}$ & $96,40 \pm 22,80^{\mathrm{b}, \mathrm{B}}$ \\
EE & 25 & 05 & $45,57 \pm 7,54^{\mathrm{a}}$ & $56,00 \pm 6,97^{\mathrm{a}, \mathrm{A}}$ \\
EE & 50 & 05 & $48,16 \pm 5,19^{\mathrm{a}}$ & $49,66 \pm 9,97^{\mathrm{a}, \mathrm{A}}$ \\
EE & 100 & 05 & $56,00 \pm 16,35^{\mathrm{a}}$ & $50,60 \pm 4,82^{\mathrm{a}, \mathrm{A}}$ \\
EE + Etanol & 25 & 10 & $45,57 \pm 7,54^{\mathrm{a}}$ & $39,54 \pm 10,52^{\mathrm{a}, \mathrm{A}}$ \\
EE + Etanol & 50 & 10 & $48,16 \pm 5,19^{\mathrm{a}}$ & $38,30 \pm 16,07^{\mathrm{a}, \mathrm{A}}$ \\
EE + Etanol & 100 & 10 & $56,00 \pm 16,35^{\mathrm{a}}$ & $47,30 \pm 15,09^{\mathrm{a}, \mathrm{A}}$ \\
EH & 25 & 05 & $47,88 \pm 14,59^{\mathrm{a}}$ & $50,40 \pm 2,05^{\mathrm{a}, \mathrm{A}}$ \\
EH & 50 & 05 & $43,12 \pm 9,26^{\mathrm{a}}$ & $67,60 \pm 9,22^{\mathrm{a}, \mathrm{A}}$ \\
EH & 100 & 05 & $41,50 \pm 4,53^{\mathrm{a}}$ & $60,33 \pm 9,03^{\mathrm{a}, \mathrm{A}}$ \\
EH + Etanol & 25 & 10 & $47,88 \pm 14,59^{\mathrm{a}}$ & $48,80 \pm 13,32^{\mathrm{a}, \mathrm{A}}$ \\
EH + Etanol & 50 & 10 & $43,12 \pm 9,26^{\mathrm{a}}$ & $42,30 \pm 9,03^{\mathrm{a}, \mathrm{A}}$ \\
EH + Etanol & 100 & 10 & $41,50 \pm 4,53^{\mathrm{a}}$ & $50,70 \pm 20,27^{\mathrm{a}, \mathrm{A}}$ \\
\hline
\end{tabular}

Letras diferentes diferem significativamente entre si $(p<0,05)$. Letras minúsculas comparam D0 com D3 na mesma linha, e letras maiúsculas comparam os tratamentos em $\mathrm{D} 3$, na mesma coluna. 
TABELA3. Níveis séricos da enzima lactato desidrogenase ( $\left(\mathrm{DH}, \mathrm{UI} \mathrm{mL}^{-1}\right.$ ) no inicio (D0) e final (D3) dos tratamentos com os extratos hexânico (EH) e etanólico (EE) das folhas de $M$. charantia sobre a atividade hepática.

\begin{tabular}{lcccc}
\hline Grupos & Dose $\left(\mathbf{m L}\right.$ ou $\left.\mathbf{~ m ~ k ~}^{-1}\right)$ & Número de Animais & D & D3 \\
\hline Salina & 0,2 & 10 & $1138,3 \pm 239,5^{\mathrm{a}}$ & $1931,8 \pm 383,64^{\mathrm{a}, \mathrm{A}}$ \\
Tween & 0,2 & 10 & $1343,0 \pm 296,2^{\mathrm{a}}$ & $1935,8 \pm 319,6^{\mathrm{a}, \mathrm{A}}$ \\
Etanol & 0,2 & 10 & $1426,3 \pm 395,2^{\mathrm{a}}$ & $1405,8 \pm 364,7^{\mathrm{a}, \mathrm{A}}$ \\
EE & 25 & 05 & $1089,5 \pm 251,9^{\mathrm{a}}$ & $1810,0 \pm 79,4^{\mathrm{a}, \mathrm{A}}$ \\
EE & 50 & 05 & $1096,1 \pm 287,2^{\mathrm{a}}$ & $1182,8 \pm 503,4^{\mathrm{a}, \mathrm{A}}$ \\
EE & 100 & 05 & $935,4 \pm 153,1^{\mathrm{a}}$ & $1124,0 \pm 234,1^{\mathrm{a}, \mathrm{A}}$ \\
EE + Etanol & 25 & 10 & $1089,5 \pm 251,9^{\mathrm{a}}$ & $1814,4 \pm 479,2^{\mathrm{b}, \mathrm{A}}$ \\
EE + Etanol & 50 & 10 & $1096,1 \pm 287,2^{\mathrm{a}}$ & $3113,4 \pm 1709,6^{\mathrm{b}, \mathrm{A}}$ \\
EE + Etanol & 100 & 10 & $935,4 \pm 153,1^{\mathrm{a}}$ & $3276,5 \pm 1531,3^{\mathrm{b}, \mathrm{A}}$ \\
EH & 25 & 05 & $1330,5 \pm 338,1^{\mathrm{a}}$ & $2337,5 \pm 441,2^{\mathrm{b}, \mathrm{A}}$ \\
EH & 50 & 05 & $1347,1 \pm 281,1^{\mathrm{a}}$ & $2126,2 \pm 542,7^{\mathrm{b}, \mathrm{A}}$ \\
EH & 100 & 05 & $1393,4 \pm 313,6^{\mathrm{a}}$ & $2287,0 \pm 376,6^{\mathrm{b}, \mathrm{A}}$ \\
EH + Etanol & 25 & 10 & $1330,5 \pm 338,1^{\mathrm{a}}$ & $1875,6 \pm 648,5^{\mathrm{a}, \mathrm{A}}$ \\
EH + Etanol & 50 & 10 & $1347,1 \pm 281,1^{\mathrm{a}}$ & $1334,3 \pm 361,2^{\mathrm{a}, \mathrm{A}}$ \\
EH + Etanol & 100 & 10 & $1393,4 \pm 313,6^{\mathrm{a}}$ & $1638,0 \pm 521,3^{\mathrm{a}, \mathrm{A}}$ \\
\hline
\end{tabular}

Letras diferentes diferem significativamente entre si $(p<0,05)$. Letras minúsculas comparam D0 com D3 na mesma linha, e letras maiúsculas comparam os tratamentos em D3, na mesma coluna.

\section{Efeito do extrato etanólico (EE) sobre a função hepática}

O pré-tratamento dos animais com EE nas três doses estudadas não impediu a elevação de AST (Tabela1), contudo preveniu o aumento de ALT provocada por etanol $(p<0,05)$ (Tabela 2). A administração de EE por três dias consecutivos não provocou elevação das aminotransferases e nem da LDH. Em D3 os níveis séricos de LDH não sofreram alterações significativas entre os diferentesgrupos (Tabela3).

Os níveis séricos de ALT demonstraram que diferentes doses de EE foram capazes de proteger 0 fígado de lesão hepatocelular ou alteração na permeabilidade da membrana celular provocada pela administração do etanol (Tabela 1).

\section{Efeito do extrato hexânico (EH) sobre a função hepática}

O pré-tratamento com EH nas três doses estudadas preveniu tanto o aumento da AST (Tabela 1) quanto da ALT induzidas pelo etanol $(p<0,05)$ (Tabela 2). Em D3 os níveis séricos de LDH não sofreram alterações significativas entre os diferentes grupos (Tabela 3), contudo o tratamento por três dias com EH provocou aumento da LDH em relação a D0.

EH demonstrou acentuada capacidade de proteção contra a agressão do etanol, reduzindo significativamente os níveis séricos de ALT em relação ao grupo que recebeu apenas etanol, mantendo os níveis séricos de ALT em D3 semelhantes a D0 (Tabela 1).

\section{DISCUSSÃO}

Embora a patogênese do álcool não seja totalmente conhecida, muitos dos efeitos tóxicos no fígado têm sido atribuídos ao estresse oxidativo. $\mathrm{O}$ etanol, ou os metabólitos, causam a auto-oxidação das células hepáticas, induzindo acentuada hepatotoxicidade, atuando como agente auto-oxidativo ou reduzindo os níveis de antioxidantes (Crawford \& Balakenhoan, 1991). A peroxidação lipídica e os danos relacionados à membrana são características chaves das injúrias hepáticas alcoólicas (Cardin et al., 2002).

As enzimas ALT e AST são consideradas hepatoespecíficas e têm aumento imediato após lesão hepatocelular ou alteração na permeabilidade da membrana celular (Ettinger \& Feldman, 1997); sendo assim funcionam como marcadores séricos (Choi et al., 2006 ).

Os extratos de plantas, sobretudo as plantas medicinais têm sido fontes atrativas de novas drogas e têm mostrado resultados promissores nos mais diversos tratamentos, inclusive como gastroprotetores e hepatoprotetores.

Neste trabalho, uma única dose de etanol provocou elevação $(p<0,05)$ dos níveis séricos de AST e ALT. O tratamento prévio com EE aumentou os níveis séricos de AST e manteve constantes os teores de ALT, enquanto EH manteve os níveis constantes tanto de AST quanto de ALT nos animais submetidos ao etanol. Dentre as transaminases, ALT é a mais específica para avaliar danos hepáticos (Giannini et al., 2005). Dessa forma, sugere-se que tanto $\mathrm{EE}$ quanto $\mathrm{EH}$, nas doses estudadas, foram capazes de proteger o fígado das lesões causadas pela administração aguda do etanol.

Flavonóides, vitaminas e sustâncias antioxidantes têm se mostrado efetivas na proteção do fígado contra os efeitos deletérios do álcool. O efeito hepatoprotetor também pode ser verificado em algumas plantas medicinais através dos níveis séricos 
enzimáticos de AST e ALT. Os extratos etanólico e aquoso de Momordica dioica Roxb. reduziram os níveis séricos de AST e ALT em lesões induzidas por tetracloreto de carbono. Esta atividade foi atribuída ao potencial antioxidante dos extratos e a presença de flavonóides (Jain et al., 2008). As folhas de Cassia auriculata demonstraram ação hepatoprotetora, mantendo os níveis séricos da ALT constantes após indução da lesão hepática por etanol (Rajagopal et al., 2003). Outra planta que também apresentou esse efeito foi a C. occidentalis (Jafri et al., 1999.). O prétratamento com o extrato de Phyllanthus emblica apresentou hepatoproteção em lesões causadas pelo etanol verificado através dos níveis séricos de AST, que se mantiveram constantes (Pramyothin et al., 2006). A utilização do extrato de caule de Acanthopanax senticosus em pré-tratamentos de lesão induzida pelo etanol também apresentou potencial de hepatoproteção (Choi et al., 2006).

O pré-tratamento com EH de folhas de $M$. charantia manteve os níveis séricos da LDH semelhantes ao D0 em animais que receberam o etanol, enquanto EE aumentou esta atividade (Tabela 3). Contudo, os animais que receberam apenas $\mathrm{EH}$ apresentaram níveis elevados desta enzima. A lactato desidrogenase (LDH) é uma enzima que se distribui amplamente pelos tecidos (Ettinger \& Feldman, 1997). Os níveis séricos dessa enzima na circulação funcionam como marcador de danos hepáticos os quais são comumente utilizados também para o diagnóstico de doenças hepáticas (Choi et al., 2006). A utilização do extrato do caule de $A$. senticosus, em pré-tratamento de lesão induzida pelo etanol, apresentou potencial de hepatoproteção, fato observado pelos níveis séricos da enzima LDH (Choi et al., 2006).

Quanto à atividade antioxidante dos extratos das folhas de $M$. charantia, verificou-se que EH teve melhor desempenho que EE. O potencial antioxidante de $\mathrm{EH}$ foi diretamente relacionado com o aumento da dose. Mediante os resultados analisados pode-se inferir que $\mathrm{EH}$ apresenta componentes que tentam manter a integridade celular, e que possivelmente estejam relacionados à elevada atividade antioxidante. Pois, tem sido relatado que a suplementação com antioxidantes promove proteção contra injúrias induzidas por etanol (Sun et al., 1999). O pré-tratamento de animais com chá verde (Augustyniak et al., 2005) bem como com extratos de raízes de Decalepis hamiltoni (Srisvastava \& Shivanandappa, 2006) e que receberam posteriormente etanol foi capaz de reduzir os níveis séricos das enzimas ALT e AST.

Conclui-se que o extrato hexânico $(\mathrm{EH})$ das folhas de $M$. charantia apresentou efeito hepatoprotetor em modelo de lesão provocada por dose única de etanol, avaliado pelos biomarcadores enzimáticos AST, ALT. Este efeito pode estar relacionado ao potencial antioxidante apresentado por $\mathrm{EH}$, demonstrando melhor desempenho neste estudo.

\section{AGRADECIMENTO}

Ao CNPq, pela bolsa de mestrado concedida à Barbara Sucupira Pereira, e ao Laboratório de Produtos Naturais da UECE coordenado pela Profa Dra Selene Maia de Morais, pelo suporte técnico.

\section{REFERÊNCIA}

ALAM, S. et al. Antiulcer activity of methanolic extract of Momordica charantia L. in rats. Journal of Ethnopharmacology, v.123, p.464-9, 2009.

AUGUSTYNIAK, A.; WASZKIEWICZ, E.; SKRZYDLEWSKA, $E$. Preventive action of green tea from changes in the liver antioxidant abilities of different aged rats intoxicate with ethanol. Nutrition, v.21, p.925-32, 2005.

BATISTA, L.M. et al. Atividade ovicida e larvicida in vitro das plantas Spigelia anthelmia e Momordica charantia contra o nematódeo Haemonchus contortus. Ciência Animal, v.9, p.67-73, 1999.

BONDET, V.; BRAND-WILLIAMS, V.; BERSET, C. Kinetics and mechanisms of antioxidant using the DPPH free radical method. Lebensm Wiss Tecnology, v.30, p.60915, 1997.

BRAGA, L.T. et al. Efeito do levamisol e do extrato etanólico de folhas de Momordica charantia sobre a dermatofitose experimental em coelhos. Ciência Animal Brasileira, v.8, n.2, p.285-95, 2007.

CARDIN, R. et al. Hepatocyte proliferation and apoptosis in relation to oxidative damage in alcohol-related liver disease. Alcohol \& Alcoholism, v.37, p.43-8, 2002.

CRAWFORD, D.W.; BALAKENHOAN, D.H. Arterial wall oxygenation oxy radicals and atherosclerosis. Atherosclerosis, v.89, p.97-108, 1991.

CHOI, J.S. et al. Glycoprotein isolated from Acanthopanax senticosus protects against hepatotoxicity induced by acute and chronic alcohol treatment. Biological \& Pharmaceutical Bulletin, v.29, p.306-14, 2006.

EL-BATRAN, S.A.E.S.; EL-GENGAIHI, S.E.; ELSHABRAWYA, O.A. Some toxicological studies of Momordica charantia L. on albino rats in normal and alloxan diabetic rats. Journal of Ethnopharmacology, v.108, p.236-42, 2006.

ETTINGER, S.J.; FELDMAN, E.C. Tratado de Medicina Interna Veterinária. 4.ed. São Paulo: Manole, 1997, v.2, 320p.

FARIAS, V.M. Modulação da resposta inflamatória por extratos de Momordica charantia em camundongos. 2003. 60p. Dissertação (Mestrado em Reprodução e Sanidade Animal) - Programa de PósGraduação em Ciências Veterinárias, Faculdade de Veterinária, Universidade Estadual do Ceará, Fortaleza. GIANNINI, E.G.; TESTA, R.; SAVARINO, V. Liver enzyme alteration: a guide for clinicians. Canadian Medical Association Journal, v.172, p.367-79, 2005.

GÜRBÜZ, I. et al. Anti-ulceratogenic effect of Momordica charantia L. fruits on various ulcer models in rats. Journal of Ethnopharmacology, v.7, p.77-82, 2000. 
JAFRI, M.A. et al. Hepatoprotective activity of leaves of Cassia occidentalis against paracetamol and ethyl alcohol intoxication in rats. Journal of Ethnopharmacology, v.66, p.355-61, 1999.

JAIN, A. et al. Antioxidant and hepatoprotective activity of ethanolic and aqueous extracts of Momordica dioica Roxb. leaves. Journal of Ethnopharmacology, v.115, p.61-6, 2008. KHAN, M.R. Momordica charantia and Allium sativum: broad-sepctrum antibacterial activity. Korean Journal of Pharmacognosy, v.29, p.155-8, 1998.

KUMAR, G.S.; SHETTY, A.K.; SALIMATH, P.V. Modulatory effect of bitter ground (Momordica charantia LINN.) on alterations in kidney heparin sulfate in streptozotocininduced diabetic rats. Journal of Ethnopharmacology, v.115, p.276-83, 2008.

KUMAR, R. et al. Fruit extracts of Momordica charantia potentiate glucose uptake and up-regulate Glut-4, PPARã and PI3K. Journal of Ethnopharmacology, v.126, p.533-7, 2009.

LEITE, K.L. et al. Efeito protetor do extrato etanólico de Momordica charantia e Lippia sidoides contra lesões gástricas induzidas experimentalmente. Ciência Animal, v.12, supl.1, p.83-7, 2002.

MATOS, F.J.A. Introdução à fitoquímica experimental. 2.ed. Fortaleza: Imprensa universitária, UFC, 1997. 139p. PLATEL, K.; SHURPALEKAR, K.S.; SRINIVASAN, K. Influence of bitter ground (Momordica charantia) on growth and blood constituents in albino rats. Die Nahrung, v.37, p.156-60, 1993.
PRAMYOTHIN, P. et al. The protective of Phyllanthus emblica Linn. extract on ethanol induced rat hepatic injury. Journal of Ethnopharmacology, v.107, p.361-4, 2006. RAJAGOPAL, S.K. et al. Activity of Cassia auriculata leaf extract in rats with alcoholic liver injury. Journal of Nutritional Biochemistry, v.14, p.452-8, 2003.

SAIRAM, K. et al. Antiulceratogenic effect of methanolic extract of Emblica officinalis: an experimental study. Journal of Ethnopharmacology, v.82, p.1-9, 2002.

SEHRAWAT, A. et al. Butea monosperma and chemomodulation: protective role against thioacetamidemediated hepatic alterations in Wistar rats. Phytomedicine, v.13, p.157-63, 2006.

SRISVASTAVA, A.; SHIVANANDAPPA, T. Heptoprotective effect of the aqueous extract of the roots of Decalepis hamiltonii against ethanol-induced oxidative stress in rats. Hepatology Research, v.35 p.267-75, 2006.

SUN, G.Y. et al. Grape polyphenols protect neurodegenetative changes induced by chronic ethanol administration. Neuroreport, v.10, p.93-6, 1999.

VIRDI, J. et al. Antihyperglycemic effects of three extracts from Momordica charantia. Journal of Ethnopharmacology, v.88, p.107-11, 2003.

VASCONCELOS, A.K.P. et al. Avaliação dos ungüentos à base de extratos hexânico ou etanólico das folhas de Momordica charantia L. sobre as lesões cutâneas experimentais em coelhos. Acta Scientiae Veterinariae, v.35, n.1, p.59-65, 2007.

Rev. Bras. PI. Med., Botucatu, v.12, n.3, p.311-316, 2010. 